\title{
SARS-COV-2: fatores associados à suscetibilidade à forma grave da covid-19
}

\section{SARS-COV-2: factors associated with susceptibility to the severe form of covid-19}

DOI: $10.46919 / \operatorname{archv2n3-010}$

Recebimento dos originais: 01/01/2021

Aceitação para publicação: 31/03/2021

\author{
Alessandro Clementino Carvalho \\ Biomedicina; Aluno de graduação em biomedicina \\ - Centro Universitário do Sul de Minas (UNIS). \\ E-mail: alessandro.carvalho@alunos.unis.edu.br \\ Priscila Henrique Moraes Paiva \\ Biomédica , Professora Doutora. \\ E-mail: priscila.paiva@professor.unis.edu.br.
}

\section{RESUMO}

Com mais de 42 milhões de casos de COVID-19, em 20 de outubro de 2020, as mortes em decorrência do SARS-CoV-2 contabilizam a marca de aproximadamente 1,1 milhão de óbitos no mundo. A elevada propagação e patogenicidade do SARS-Cov-2, a inexistência de um tratamento efetivo e a imunização preventiva geram instabilidade política, financeira e psicológica mundialmente. Apesar disso, inúmeros estudos têm sido realizados no intuito de compreender melhor a doença, sobretudo os fatores que predispõem a sua forma grave. Desse modo, o presente artigo tem como propósito realizar uma revisão de literatura de pesquisas publicadas recentemente sobre os elementos relacionados à suscetibilidade no desencadeamento da forma grave da COVID-19. Sabe-se que aproximadamente $15 \%$ dos pacientes infectados pelo vírus necessitam de cuidados especiais e como consequência a internação. Apesar de cada indivíduo possuir uma resposta imunológica diferente perante a patologia, fatores como grupo sanguíneo, presença ou não de comorbidades, características genéticas associadas ao sexo, elevada expressão do gene ACE-2 e até tolerâncias distintas a falta de oxigênio serão abordados e discutidos nessa revisão, proporcionando contribuições para a comunidade acadêmica em desenvolvimento de ações com embasamento científico responsável.

Palavras-chave: Suscetibilidade, Covid-19, Gravidade.

\begin{abstract}
With over 42 million cases of COVID-19, as of October 20, 2020, deaths as a result of SARS-CoV-2 account for the mark of approximately 1.1 million deaths worldwide. The high spread and pathogenicity of SARS-Cov-2, the lack of an effective treatment and preventive immunization create political, financial, and psychological instability worldwide. Despite this, numerous studies have been conducted in order to better understand the disease, especially the factors that predispose to its severe form. Thus, the purpose of this article is to conduct a literature review of recently published research on the elements related to susceptibility to the onset of the severe form of COVID-19. It is known that approximately $15 \%$ of patients infected with the virus require special care and, as a consequence, hospitalization. Although each individual has a different immune response to the pathology, factors such as blood group, presence or absence of comorbidities, genetic characteristics associated with gender, high expression of the ACE- 2 gene and even different tolerances to lack of oxygen will be addressed and discussed in this review, providing contributions to the academic community in developing actions with responsible scientific basis.
\end{abstract}


Keywords: Susceptibility, Covid-19, Severity.

\section{INTRODUÇÃO}

A forma grave da COVID-19 se apresenta como um dos pilares de estudo elencados com a crise de saúde pública que o mundo evidencia por meio do colapso nos estabelecimentos de saúde e os impactos diretos na sociedade. Porém, a manifestação da patologia severa está relacionada com danos definitivos no indivíduo acarretando muitas das vezes o caso clínico de óbito. Deste modo, a atual questão vem alcançando evidência pelos pesquisadores e profissionais de saúde através da prática vivenciada na clínica. Com isso os estudos buscam como objetivo manter o entendimento da distribuição de casos severos nas diferentes populações, bem como os fatores relacionados com este desencadeamento para com a finalidade de preservar a saúde destas pessoas e contribuir para o fim da pandemia vigente.

Todas as pessoas estão sujeitas a contrair a COVID-19, levando em consideração a alta transmissibilidade e as rotas de transmissão viral. Porém, nem todas as pessoas estão sujeitas a desenvolver uma patologia mais severa. A forma grave da COVID-19 provém de fatores individuais e intrínsecos e depende da capacidade imunológica de cada pessoa frente à infecção. As pessoas que apresentam uma fisiopatologia branda, em suma, não necessitam do auxílio médico, porém os quadros moderados a grave, devem buscar ajuda médica desde o surgimento das primeiras ocorrências, visto que o prognóstico prévio está totalmente relacionado ao monitoramento e restrição da infecção.

O presente trabalho adota como metodologia uma revisão de artigos já existentes na literatura desde o surgimento dos primeiros casos da patologia até o término do ano da publicação.

Portanto, a grande questão deste estudo é contemplar os aspectos relacionados com a suscetibilidade à forma grave da COVID-19 e entender a relação existente entre a gravidade da doença com a relação indivíduo-hospedeiro. À medida que se ganha campos na área do conhecimento frente ao SARS-CoV-2, estas descobertas estão diretamente relacionadas com o diagnóstico e a terapêutica.

\section{FISIOPATOLOGIA}

A assertiva que leva em consideração a gravidade da COVID-19, está relacionada com além dos fatores de risco estabelecidos (obesidade, comorbidades, prática de atividade física, tabagismo, entre outros), os fatores individuais de cada ser, estes fatores transcrevem a suscetibilidade a forma grave da patologia (GRANDY et al., 2020). A vulnerabilidade à forma grave da COVID-19 é uma importante preocupação pública e se infere a necessidade de acender o manejo de políticas públicas de urgência para o controle e planejamento da assistência e saúde em áreas críticas (SANTOS et al., 2020). 
Embora a COVID-19 não possua uma letalidade elevada se comparada com outras doenças de sua mesma família como MERS-CoV e SARS-CoV, sua característica transmissibilidade a indivíduos pertencentes a grupos de risco e/ou com presença de comorbidades eleva sua letalidade consideravelmente (MOREIRA, 2020). A letalidade aumenta em 10,5\% para doenças cardiovasculares, 7,3\% na diabetes, 6,3\% no caso de doenças respiratórias crônicas e 6\% para portadores de hipertensão arterial sistêmica (WU; MCGOOGAN, 2020).

Referente ao coronavírus podemos observar o tropismo pelas células da mucosa do trato respiratório. A ECA2 (enzima conversora de angiotensina 2) é um fator crucial para a posterior ligação que permite que o vírus entre na célula e desenvolva todos os sintomas e o desencadear da patologia (LUKASSEN, 2020). A entrada viral e a presença concomitante do vírus alavancam a inflamação e acúmulo de líquidos, o que gera um dos sintomas que representa a maior procura médica: a dificuldade respiratória (CARNEIRO; DALLIGNA, 2020).

O SARS-CoV-2 possui em sua superfície uma proteína que recebe o nome de proteína S. É através do reconhecimento da proteína S pelo receptor ECA-2 presente no hospedeiro humano, que acontece o início da patologia. Posteriormente o vírus realiza a replicação através do tecido epitelial mucoso que compõe o trato respiratório e nas células alveolares. A resposta imunológica é benéfica para a longo prazo criar imunidade específica, porém pode contribuir para o agravamento quando em excesso (SILVA et al., 2021).

Os sintomas da COVID-19 não se restringem apenas a extensão do trato respiratório, podendo abranger os diversos órgãos que compõem o organismo humano (GOUDOURIS, 2021). A patologia severa, necessita de maior cuidado clínico, seja pela necessidade de ventilação mecânica ou pela demanda de unidades de terapia intensiva (TAGARRO et al., 2020). Os danos envolvem os tecidos que dispõe da presença da enzima conversora de angiotensina 2 (ECA-2) (ALVES et al., 2021) e a forma grave intercorre a presença de danos na extensão do trato respiratório, tecido cardiovascular, tecido hepático, tecido renal, tecido gastrintestinal, entre outros (SUÁREZ et al., 2021).

Por meio da ação viral na região respiratória contribuindo para danos subsequentes as células que compõem os alvéolos pulmonares e a defesa imunológica exíguo, estes eventos colaboram para a perda da capacidade de trocas gasosas e a sensação de hipoventilação adjunta a falta de ar, quadro comum na patologia severa (NSANZABERA, 2021). A coagulação intravascular disseminada (CID) é um quadro comumente relacionado com a gravidade da doença, especialmente em estudos que utilizam os marcadores: dímero-d e produto de degradação de fibrina, sendo que demonstram sua correlação com os óbitos da COVID-19 (TANG et al., 2020).

A grande maioria dos pacientes conseguem conter o coronavírus apresentando uma doença leve e autolimitada com um ótimo prognóstico com apenas sinais considerados leves, que são a perda de paladar 
e/ou olfato (pela ação e replicação viral naquela região), dor de garganta, congestão nasal, mal estar, febre, tosse seca, diarreia, vômitos e dor muscular. O tratamento neste momento é feito pelo alívio sintomático (CASCELLA, 2020).

São os sintomas moderados e graves que está relacionado com a procura de auxílio médico (MACINKO et al., 2020), principalmente pelo vírus atingir o trato respiratório inferior causando comprometimento respiratório, acometimento pulmonar com a replicação viral resultante no edema pulmonar e pôr fim a inflamação localizada. Algumas complicações paralelas como a sepse e choque séptico estão associados com uma disfunção orgânica com o risco de vida e da mortalidade pelo presente quadro clínico (PÉREZ, CORDEIRO, AVENDANÕ, 2020).

\section{FATORES ASSOCIADOS A FORMA GRAVE DA COVID-19}

A tabela 1 resume os principais fatores descritos e associados a forma grave da doença

\begin{tabular}{|c|c|c|}
\hline Fatores & Manifestações clínicas & Referências \\
\hline Envelhecimento & $\begin{array}{l}\text { Imunodepressão, resposta } \\
\text { descompensada, aumento de citocinas pró- } \\
\text { inflamatórias, diminuição da resposta-vacina e por } \\
\text { fim aumento da expressão da ACE-2, desencadeando } \\
\text { a forma grave da COVID- } 19 \text {. }\end{array}$ & $\begin{array}{l}\text { (VELAVAN; MEYER, } \\
\text { 2020). } \\
\text { (CHEN et al., 2020). } \\
\text { (YUKI; FUJIOGI; } \\
\text { KOUTSOGIANNAKI, } \\
\text { 2020). } \\
\text { (TAVARES et al., 2020). }\end{array}$ \\
\hline ACE-2 & $\begin{array}{l}\text { Receptor humano, distribuindo em toda a via } \\
\text { respiratória e diversas partes do corpo, responsável } \\
\text { pela função de ligação SARS-CoV-2 (Spike) e corpo } \\
\text { humano. }\end{array}$ & $\begin{array}{l}\text { (OUDIT et al., 2009). } \\
\text { (XU et al., 2020). }\end{array}$ \\
\hline Tabagismo & $\begin{array}{l}\text { Diminuição na proteção do epitélio de revestimento, } \\
\text { deprime a função de célula relacionadas com o } \\
\text { sistema imunológico, maior concentração da ACE-2, } \\
\text { aumento na severidade da doença e complicações } \\
\text { respiratórias. Fator de gravidade para a doença } \\
\text { severa. }\end{array}$ & $\begin{array}{l}\text { (FELDMAN; } \\
\text { ANDERSON, 2013). } \\
\text { (BRAKE et al., 2020). }\end{array}$ \\
\hline Hipertensão & $\begin{array}{l}\text { Aumento da expressão da ACE- } 2 \text { e caminha com } \\
\text { comorbidades secundárias que são fator de risco para } \\
\text { a COVID-19. Fator de gravidade para a doença } \\
\text { severa. }\end{array}$ & (WAN et al., 2020). \\
\hline Diabetes & $\begin{array}{l}\text { Facilita a tempestade de citocinas, devido a } \\
\text { inflamação crônica e de baixo grau, aumento } \\
\text { significado de Interleucina-6, aumentando a resposta } \\
\text { inflamatória descontrolada e prejudicando a função } \\
\text { das células T. Fator de gravidade para a doença } \\
\text { severa. }\end{array}$ & $\begin{array}{l}\text { (MADDALONI; } \\
\text { BUZZETTI, 2020). }\end{array}$ \\
\hline Obesidade & $\begin{array}{l}\text { Acarreta a chegada de comorbidades secundárias que } \\
\text { são fator de risco para a COVID-19, pacientes } \\
\text { acometidos apresentam quadros clínicos } \\
\text { desafiadores, diminuição na saturação de oxigênio, } \\
\text { inflamação de baixo grau, secreção anormal de } \\
\text { imunomarcadores. Fator de gravidade para doença } \\
\text { severa. }\end{array}$ & $\begin{array}{l}\text { (SIMONNET et al., } \\
2020) \text {. }\end{array}$ \\
\hline
\end{tabular}


Lesão Renal

DPOC*

DPV*

Gênero

ApoE

HLA*
Desequilíbrio nos fluidos corporais, aumento da carga viral, liberação de nefro toxinas, aumento de enzimas, incidência de quadros de hipercoagulação resultante as doenças renais. Fator de gravidade para a doença severa.

Maior expressão de ACE-2, necessidade de ventilação mecânica, maior secreção de muco.

Elevação da mortalidade. Pode resultar em pacientes acometidos pela COVID-19: Frequência cardíaca anormal, danos ao miocárdio, síndrome coronariana aguda e tromboembolismo.

Grupo de vulnerabilidade, propensão em desenvolver a forma grave devido a imunodepressão presente nos tratamentos da patologia.

Sexo masculino maior número de quadros severos e óbitos frente a COVID-19, visto que a resposta imunológica feminina é maior, além do hormônio testosterona estar ligado a inflamação.

A chance de contrair a COVID-19 e sua progressão a severidade é um platô nos grupos sanguíneos A e diminuída nos grupos sanguíneos O. Grupo sanguíneo A: Fator de Willebrand 25\% aumentado.

Grupo sanguíneo B e O: A presença do anti-a em sua estrutura promovem um efeito protetor intrínseco. HLA-B * 46: 01 risco aumentado para a infecção, frente a COVID-19.

HLA-A * 11: 01, B * 51: 01 e C *14: 02 incidências do quadro grave. Fator de doença severa.

Indivíduos que expressam de maneira duplicada a ApoE-4 possuem maiores chances de desencadear a forma grave da COVID-19.

ApoE4: 2,2 vezes mais de probidade de possuírem a doença grave e 4,3 chances a mais de morrer de COVID-19 em detrimento aos que expressam ApoE3 .
(RONCO; REIS; SYED, 2020).

(CARDOSO, 2020).

(NISHIGA et al., 2020).

(KAWAHARA et al., 2020).

(KLEIN; MARRIOTT; FISH, 2015)

(CANTERO, 2021)

(JAILLON;

BERTHENET

GARLANDA, 2019).

(ZIETZ; ZUCKER;

TATONETTI, 2020).

(GUILLON et al., 2008).

(ANASTASSOPOULOU

et al., 2020).

(WANG et al., 2020).

(KUO et al., 2020).

*DPOC: doença pulmonar obstrutiva crônica; DPV Doença vascular periférica, HLA: antígeno leucocitário humano

\subsection{ENVELHECIMENTO}

A inflamação é um evento essencial para responder o dano tecidual e limpeza tecidual, porém nos casos de COVID-19 amenizar a hiper inflamação pode melhorar a resposta imunológica frente a patologia (AKBAR; GILROY, 2020). As pesquisas existentes na literatura esclarecem que a alta faixa etária e a imunodepressão está diretamente relacionada com a suscetibilidade à forma grave da doença, sendo um fator de risco possível (VELAVAN; MEYER, 2020).

O aumento da resposta inflamatória contribui também para o agravamento da patologia, visto que muitos autores associam o conceito de "tempestade inflamatória e tempestade de citocinas" (VIEIRA et al., 2020). A hiper inflamação sistêmica na COVID-19 tem associação direta com o aparecimento de quadros de trombose, CID (coagulação intravascular disseminada) e a exaustão de células do sistêmica imunológico como os linfócitos (linfopenia) (BLAGOSKLONNY, 2020). 
Figura 1 - Tempestade de citocinas com relação na hiperfunção e exaustão celular.

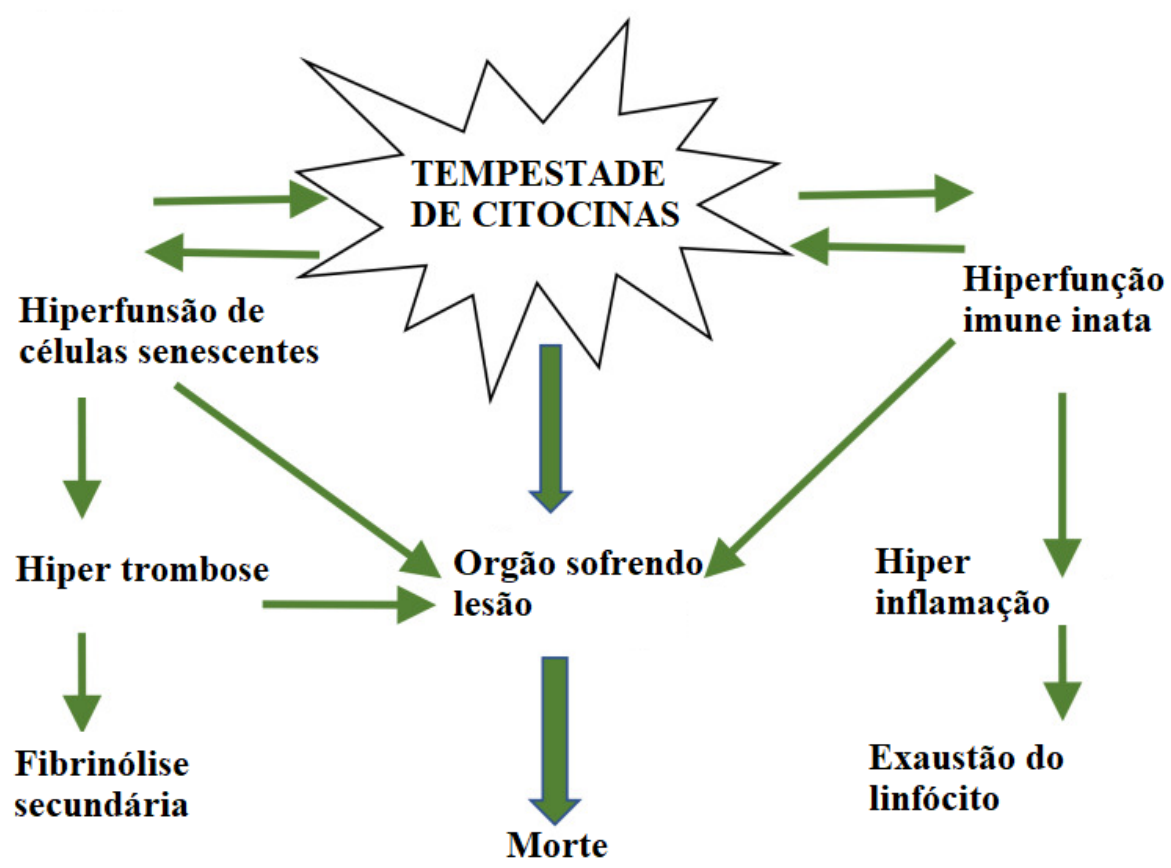

Fonte: BLAGOSKLONNY, 2020.

O envelhecimento é um processo fisiológico natural. Este processo colabora como fator de risco consagrado para a gravidade da COVID-19 e para diversas doenças, existe uma relação do envelhecimento com a posterior desregulação da resposta imune (CHEN et al., 2020). Há uma especulação que se baseia no aumento concomitantemente de citocinas que por sua vez aumentam consideravelmente as respostas pró-inflamatórias como exemplificado na ARDS (Síndrome de angústia respiratória do adulto), porém espera-se a existência de estudos em animais futuros que possam comprovar tal hipótese na COVID-19 (YUKI; FUJIOGI; KOUTSOGIANNAKI, 2020).

As diferenças na competência imunitária em alusão ao envelhecimento, colaboram também para que os idosos detenham maior concentração de cargas virais (visto que o corpo não possui competência de controlá-la) e ao mesmo tempo haja uma persistência do agente viral. Os pacientes de idade avançada têm produção de anticorpos diminuída e uma posterior relação com a diminuição na intensidade da respostavacina (TAVARES et al., 2020).

A enzima conversora de angiotensina 2 tem a função de receptor humano para o SARS-CoV-2 (OUDIT et al., 2009). Este receptor, se encontra distribuído no epitélio das vias respiratórias, bem como a entrada viral depende dessas células alvo e podem fornecer possíveis rotas de infecção dentro do organismo (XU et al., 2020). Conforme o envelhecimento gradativo natural do corpo humano, há um aumento constante na expressão de ACE-2, que determina um aumento considerável na capacidade do organismo se infectar, bem como a severidade frente ao coronavírus. Embora seja um fator importante ele não é o único capaz de mudar a fisiopatologia da doença (ANDRADE et al., 2020). 


\subsection{TABAGISMO}

O consumo de tabaco tem aumentado consideravelmente na população adolescente em sua maioria compostos de tabaco que detém a presença de aromatizantes/aditivos. O tabaco se apresenta como um dos principais fatores associados ao surgimento de doenças crônicas não transmissíveis (CAMPOS; SOUZA, 2018). Embora esteja associado a desencadear a forma grave da COVID-19, a obesidade e o tabagismo se apresentam como fatores de risco evitáveis, sendo uma preocupação de saúde pública, o tabagismo é responsável por 7 milhões de óbitos anualmente (SALES et al., 2019).

O tabaco se apresenta como fator de risco para desenvolvimentos de grande parte das infecções orais e respiratórias, bem como o manejo de sua gravidade. Isso porque ele e seus derivados transcrevem uma diminuição na proteção do epitélio que reveste as vias aéreas, deprime a função de células como: macrófagos alveolares, células dendríticas, células natural killer e neutrófilos (FELDMAN; ANDERSON, 2013).

Pacientes tabagistas apresentam uma maior concentração da ACE2 em resposta ao processo inflamatório decorrente das substâncias presentes no cigarro no trato respiratório, portando uma maior concentração desta enzima está relacionada com a progressão para complicações respiratórias graves nesses pacientes (BRAKE et al., 2020).

\subsection{MÚLTIPLAS COMORBIDADES}

A presença de comorbidades existentes, é um fator crucial para a elevação da gravidade da COVID19 (MACIEL et al., 2020). Diversas pessoas não possuem o conhecimento da sua comorbidade ou de certa forma nunca realizaram um diagnóstico, fator interessante que relaciona este processo como comorbidades silenciosas, visto que sua permanência e a infecção pela COVID-19 concomitantemente se apresentam como um desfecho desfavorável da infecção pelo coronavírus (SOSIBO; KHATHI, 2020).

É viável observar que os indivíduos com doenças crônicas estabelecidas com quadros de hipertensão arterial, diabetes, doenças cardiovasculares, insuficiência renal e de idade avançada transcrevem uma maior recorrência a necessidade de procura médica hospitalar, bem como a necessidade de equiparação de amparo médico e ventilação mecânica (GAO et al., 2020).

A hipertensão é uma doença amplamente distribuída no mundo todo, sendo um problema de saúde pública intrínseco da sociedade contemporânea. O quadro clínico de hipertensão muitas das vezes é subestimado pelos pacientes e no caso da COVID-19 pode causar um aumento considerável na expressão da ACE-2, além de ser uma doença silenciosa e caminhar com outras comorbidades subjacentes (WAN et al., 2020).

Nos diversos estudos que pluralizam a diabetes ao coronavírus, podemos observar danos deletérios pela presença desta doença. A diabetes por sua vez se apresenta como um dos principais contribuintes para 
a gravidade da COVID-19, devido propiciar uma inflamação crônica de baixo grau e prolongada que acaba facilitando a tempestade de citocinas. A diabetes desencadeia um aumento de Interleucina- 6 que mensura um papel importante na facilitação da resposta inflamatória descontrolada de pacientes com COVID-19, que por sua vez alteram o quadro da doença, e prejudica a função das células T (MADDALONI; BUZZETTI, 2020).

A obesidade pode acarretar a chegada de doenças secundárias de caráter crônico, como hipertensão, diabetes do tipo II, doenças cardiovasculares, entre outros. Estas doenças também se apresentam como fator de risco para a COVID-19. A obesidade transcreve desafios e quadro clínico mais desafiador devido aos prejuízos na ventilação da base dos pulmões, diminuição da saturação do oxigênio do sangue, inflamação de baixo grau que prejudica as capacidades imunológicas, secreção anormal de imunomarcadores como adipocinas, interferons e citocinas que prejudicam a homeostasia celular (SIMONNET et al., 2020).

A Lesão renal aguda está presente em pacientes gravemente afetados pela COVID-19, sua alusão com o coronavírus depende de diversos fatores como desequilíbrio nos fluidos corporais, aumento da carga viral, liberação de nefrotoxinas, aumento de enzimas como a dipeptidil peptidase-4 (BATTLE et al., 2020), que funciona como um sub-receptor para uma subunidade do coronavírus, bem como quadros de hipercoagulação resultante de doenças renais (RONCO; REIS; SYED, 2020).

Pessoas acometidas por Doença Pulmonar Obstrutiva Crônica (DPOC), possuem uma taxa mais elevada de receptores de ACE-2, uma maior necessidade de ventilação mecânica na assistência médica e maior secreção de muco (CARDOSO, 2020). O IFN- $\lambda$ (lambda), possui importante papel no equilíbrio imunológico inato, sendo secretado rapidamente posterior a infecção viral. No caso da asma existe uma desregulação na secreção desta citocina, afetando diretamente a capacidade imunológica contra o coronavírus (CONTOLI et al., 2006).

A taxa de letalidade de pacientes infectados pela COVID-19 acometidos com Doença Vascular Periférica (DPV) demonstram que as doenças cardiovasculares elevam a mortalidade pelo coronavírus, em detrimento a qualquer outra comorbidade (incluindo doenças respiratórias crônicas ou doenças malignas). A patogênese da COVID-19 em um paciente com este quadro pode resultar em frequência cardíaca anormal, danos ao miocárdio, síndrome coronariana aguda, e tromboembolismo, diretamente relacionada com o óbito em pacientes hospitalizados (NISHIGA et al., 2020).

Pacientes portadores de doenças malignas ativas ou com caráter de remissão desenvolvem no contexto da COVID-19 um importante grupo de risco, sendo a vulnerabilidade em hospitais, propensão em desenvolver a forma grave, o tratamento da patologia transmitir quadros de imunodepressão ao paciente facilitando o avanço pelo SARS-CoV-2, taxas mais acentuadas de óbito (KAWAHARA et al., 2020). 


\subsection{GÊNERO}

O aspecto referente ao sexo é importante, pois além de fazerem alusão as diferenças genéticas entre os sexos, tangem ao sexo masculino o maior número de óbitos referente a COVID-19, uma maior gravidade na patologia e uma menor procura do serviço de saúde (ORELLANA et al., 2020). Vale ressaltar também que padrões como prevalência do tabagismo, é mais presente no sexo masculino, explicando, portanto, tal explanação (VÁZQUEZ; RIPOLL, 2020).

O dimorfismo entre os gêneros feminino e masculino está relacionado com a herança cromossômica, sendo os homens haploides na presença do cromossomo $\mathrm{X}$ e as mulheres responsáveis por receber diploidia, sendo XX. Esta diferença elenca o alargamento da resposta imunológica da mulher, visto que vários genes presentes no cromossomo $\mathrm{X}$ codificam uma associação forte com a resposta imunológica (JAILLON; BERTHENET; GARLANDA, 2019).

Além da capacidade imunológica aumentada do sexo feminino, este mesmo gênero apresenta maior aparato de anticorpos com a função de memória, bem como uma maior presença de reações adversas perante a aplicação da vacina e por fim o hormônio masculino testosterona é relacionada com a diminuição de citocinas advindos anteriormente a inflamação (KLEIN; MARRIOTT; FISH, 2015).

A presença de um gene denominado TLR7 é capaz de codificar uma proteína humana que apresenta diversas funções, entre elas sensibilidade endossômica bem como a capacidade de reconhecer RNA de fita simples. No caso da COVID-19, foi mensurado que a presença desta proteína é majoritariamente aumentada em mulheres, bem como sua expressão tem forte relação a resistência a infecções virais (CONTI; YOUNES, 2020).

A herança genética do cromossomo X, presença de hormônios sexuais que diferem os sexos, exposição a determinados riscos, diferenças de capacidades imunitárias (CANTERO, 2021), são uma das formas de explicar esta cadência de dados de que embora a pandemia afete ambos os sexos, os homens em diversos dados epidemiológicos estão mais envolvidos com uma maior taxa de mortalidade e necessidade iminente de hospitalização (SOUZA; RANDOW; SIVIERO, 2020).

\subsection{FATORES GENÉTICOS}

\subsubsection{Grupos sanguíneos}

O coronavírus também infecta células que revestem os vasos sanguíneos que circundam a região pulmonar causando pequenos danos na parede dos vasos sanguíneos. Em resposta a este dano o corpo libera uma quantidade maior de proteínas que estimulam a agregação de plaquetas que se agrupam no local da lesão. Dependendo do tamanho e profundidade dos vasos sanguíneos isso pode favorecer o surgimento de tromboses (REIS; LIMA, 2020). 
Existe uma relação proposta por diversos cientistas relacionando a existência de um conjunto de genes identificados como 3p21.31 como um lócus de suscetibilidade genética em pacientes portadores da patologia com o quadro de insuficiência respiratória e é, portanto, afirmado a correlação potencial dos grupos sanguíneos (ELLINGHAUS et al., 2020).

Encontrado estas evidências foi evidenciado que as chances de contrair a COVID-19 e a sua possível progressão é um platô nos grupos sanguíneos A e diminuída nos grupos sanguíneos $\mathrm{O}$, o que entra de acordo com estudos realizados no início da pandemia nos epicentros de Wuhan e Schenzen (ZIETZ; ZUCKER; TATONETTI, 2020).

Há muito tempo a ciência estabeleceu ao grupo sanguíneo não $\mathrm{O}$ uma maior vulnerabilidade de risco de doenças vasculares periféricas e arteriais, isquemias cerebral, tromboembolismo venoso e infarto agudo do miocárdio, entre outras doenças de caráter infeccioso (WU et al., 2008). Esta vulnerabilidade é descrita pelo fato que o grupo sanguíneo $\mathrm{ABO}$ influencia os níveis circulantes da glicoproteína de funções coagulatória chamada fator de von Willebrand. Este caráter genético determina que o fator de von Willebrand é 25\% mais elevado em indivíduos com sangue não O. Esta glicoproteína desempenha funções importantes no equilíbrio da função sanguínea como a interação entre plaquetas no local lesado e o transporte de moléculas como o fator anti-hemofílico (JENKINS; DONNELL, 2006).

No caso do SARS-CoV, foi mensurado que a ligação da proteína Spike com o receptor humano ACE-2 foi inibida pela ação de anticorpos anti-A do sangue, presentes nos tecidos sanguíneos do tipo B e tipo O. Estes estudos promovem o efeito protetor intrínseco genético do sistema ABO, esta característica estrutural do $\mathrm{ABO}$ poderia ser uma vantagem de estudo e de esclarecer a diminuição da transmissão do coronavírus na presente população (GUILLON et al., 2008).

\subsubsection{HLA (Antígeno Leucocitário Humano)}

O HLA é um gene localizado no cromossomo 6, o qual compreende uma relação de processos importantes no reconhecimento e apresentação de antígenos próprios ou externos para as células $\mathrm{T}$ do organismo. Este mecanismo preenche uma capacidade importante para o sistema imunológico adaptativo frente a determinados antígenos (GOLDBERG; RIZZO, 2015). No caso da COVID-19 células com a função de apresentar o antígeno fagocitam o SARS-CoV-2, degradam o agente viral em partículas menores e são carregados pelo MHC, até apresentar para as células T e seguir as etapas consecutivas da resposta imunológica (TAYLOR; BOLTON; BRADLEY, 2011).

Pensando na função do gene HLA, pesquisadores em genética imunológica estão investigando como acontece a resposta imunológica frente a COVID-19. Durante o processo de resposta imunológica foi elucidado que um grupo de pacientes que detinham a doença, o gene HLA se ligava em maior frequência na proteína $\mathrm{N}$ e com menor frequência na proteína $\mathrm{S}$, uma proteína importante na entrada do patógeno e sua 
sobrevivência dentro do organismo humano. Entender essa compensação é de suma importância para determinar porque determinadas populações possuem quadros mais severos em detrimento a outras, citando o modelo da população afrodescendente, residente nos Estados Unidos (INCA, 2021).

Durante o reconhecimento do SARS-CoV-2, diversas classes da molécula do HLA se ligam nas projeções do agente viral. No caso, a diversidade existente dos alelos HLA traduzem importâncias clínicas significativas. Cabe ao HLA-B * 46: 01, presente no organismo uma menor capacidade ligante ao SARSCoV-2 e um risco aumentado para a infecção frente a COVID-19, em contrapartida o HLA-B * 46: 01, ao apresentar o antígeno, esta molécula preserva em maior quantidade os peptídeos estruturais do SARS-CoV2 (ANASTASSOPOULOU et al., 2020). A capacidade ligante dos três alelos HLA-A * 11: 01, - B * 51: 01 e - C * 14: 02 tem significado perante a severidade da patologia e transcrevem uma suscetibilidade do organismo humano em enfrentar o agente viral e manifestar o quadro grave (WANG et al., 2020).

\subsubsection{Apolipoproteína E (ApoE)}

A apolipoproteína E (ApoE), pertencente no organismo humano, compõe uma classe de apolipoproteínas que possuem funções de transporte e metabolismo de lipídios, bem como o catabolismo de lipídios no sistema nervoso central e exteriormente a ele. É formada pelos lipócitos, células parenquimatosas do fígado, astrócitos cerebrais e macrófagos. Estas proteínas apresentam três formas importantes denominadas por Apo E2, E3 e E4, resultante dos alelos e2, e3, e4 do cromossomo 19, correspondente pela região 19q13.32 (VALLADARES; PERDOMO; PABÓN, 2006).

Diversas associações foram propostas após o estudo desta estrutura proteica, inicialmente ela foi associada a doenças de caráter obstrutivo dos vasos sanguíneos e doenças cardiovasculares (SING; DAVIGNON, 1985). Posteriormente os estudos apontaram a recorrência desta proteína associada a doença de Alzheimer, explicando os pacientes de idade avançada com lucidez durante toda a sua vida em detrimento a outros pacientes jovens, afligidos por problemas mentais (CORDER et al., 1994).

Em virtude, estudos recentes apontam que indivíduos que possuem expressão duplicada de ApoE4, possuem maiores chances de desencadear a forma grave da COVID-19 e levarem a óbito, do que os que expressam ApoE-3 independente de fatores anteriormente elucidados, tais quais demência ou doenças cardiovasculares, além de no futuro induzirem a maior propensão de sintomas neurológicos. Dentre os participantes envolvidos na pesquisa, os indivíduos que detinham ApoE4, possuíam aproximadamente 2,2 vezes mais possibilidade de possuírem a doença grave, bem como os dados estimam que possuem 4,3 chances a mais de morrer de COVID-19 ao invés dos indivíduos que expressam a ApoE3 (KUO et al., 2020). 


\section{CONSIDERAÇÕES FINAIS}

Existem diversos fatores associados à gravidade da COVID-19, esta patologia se apresenta multifatorial. Os fatores podem ser classificados como intrínsecos de herança genética e não mutáveis (grupo sanguíneo, condições genéticas, receptores, gênero sexual) ou podem ser fatores adquiridos conforme o estilo de vida (envelhecimento, tabagismo, sedentarismo, comorbidades adquiridas).

Compreende-se então que elucidar os fatores que transcrevem a suscetibilidade à forma grave da COVID-19 separaria grupos de maior vulnerabilidade que apresentam mais risco recorrente e que detenham uma maior propensão a desenvolver um quadro insatisfatório. Entender como o coronavírus afeta as diferentes populações, auxiliam a interpretar porque alguns pacientes tem um prognóstico bom e autolimitado e quais necessitam de uma assistência intensiva, direcionar a uma terapêutica personalizada levando em conta o curso da patologia.

Embora diversos fatores foram mencionados neste estudo, alguns deles ainda não estão bem estabelecidos e a comunidade científica carece de estudá-los a fundo, reforçando os dados e demais variáveis, sendo este tema de suma importância para as contribuições na decorrente pandemia da COVID19. 


\section{REFERÊNCIAS BIBLIOGRÁFICAS}

AKBAR, Arne N.; GILROY, Derek W. Aging immunity may exacerbate COVID-19. Science, v. 369, n.6501, p. 256-257, 2020.

ALVES, Amanda Mandarino; YVAMOTO, Erika Yuki; MARZINOTTO, Maira Andrade Nacimbem; TEIXEIRA, Ana Cristina de Sá; CARRILHO, Flair José. Braz. J. Infect. Dis., v.24, n.6, 2021.

ANASTASSOPOULOU, Cleo; GKIZARIOTI, Zoi; PATRINOS, George P; TSAKRISS, Athanasios. Human genetic factors associated with susceptibility to SARS-CoV-2 infection and COVID-19 disease severity. Hum Genomics, v.14, n.40, 2020.

ANDRADE, Pedro Mafra; BARBOSA, Samuel Cavalcante Souza; Malta, Voney Fernando Mendes; Silva, Paulo Henrique Alves; Malta, Rafael Wanderley Persiano. The variation of ACE2 expression influence in the severity of COVID-19 in adults and children: a systematic review. Braz. Ap. Sci. Rev, v.4, n.6, p. 37923804, 2020.

BATTLE, Daniel; SOLER, Maria Jose; SPARKS, Matthew A.; HIREMATH, Swapnil; SOUTH, Andrew M.; WELLING, Paul A. Acute Kidney Injury in COVID-19: Emerging Evidence of a Distinct Pathophysiology. Journ. of the Amer. Socie. of Neph., v.31, n.7, p. 1380-1383, 2020.

BLAGOSKLONNY, Mikhail V. From causes of aging to death from COVID-19. Journ. Albany NY., v.12, n.11, 2020.

BRAKE, Samuel James; BARNSLEY, Kathryn; LU, Wenying; MCALINDEN, Kielan Darcy; EAPEN, Mathew Suji; SOHAL, Sukhwinder Singh. Smoking Upregulates Angiotensin-Converting Enzyme-2 Receptor: A Potential Adhesion Site for Novel Coronavirus SARS-CoV-2 (Covid-19). J. Clin. Med., v.9, n.3, p. 841,2020 .

CAMPOS, Maryane Oliveira; SOUZA, Maria de Fátima Marinho. Fatores de risco e proteção para as doenças crônicas não transmissíveis em adolescentes nas capitais brasileiras. Rev. bras. epidemiol., v.21, n.1, 2018.

CANTERO, María Teresa Ruiz. Health statistics and invisibility by sex and gender during the COVID-19 epidemic. Gaceta Sanitaria, v.35, n.1, p. 95-98, 2021.

CARDOSO, Alexandre Pinto. A DPOC e o COVID-19. Pulmão RJ, v.29, n.1, p. 43-46, 2020.

CARNEIRO, Audrey Brascher Carneiro; DALLIGNA, Dhébora Mozena. Obesidade como fator de risco para o agravamento da Covid-19:uma breve revisão de literatura. Vitalle, v.32, n.3, p.12-18, 2020.

CASCELLA, Marco; RAJNIK, Michael; CUOMO, Arturo; DULEBOHN, Scott C; NAPOLI, Raffaela Di. Features, Evaluation and Treatment Coronavirus (COVID-19). StatPearls, 2020.

CHEN, Yiyin; KLEIN, Sabra L.; GARIBALDI, Brian T.; LI, Huifen; WU, Cunjin; OSEVALA, Nicole M.; LI, Taisheng; MARGOLICK, Joseph B.; PAWLEC, Graham; LENG, Sean X. Aging in COVID-19: Vulnerability, immunity and intervention. Ageing Research Reviews, v.65, 2021.

CONTI, P. YOUNES, A. Coronavirus COV-19/SARS-CoV-2 affects women less than men: clinical response to viral infection. J. Biol. Regul. Homeost. Agents., v.34, n.2, p. 339-343, 2020. 
CONTOLI, Marco. MESSAGE, Simon D; STANCA, Vasile Laza; EDWARDS, Michael R; WARK, Peter A. B; BARTLETT, Nathan W; KEBADZE, Tatiana; MALLIA, Patrick; STANCIU, Luminita A; PARKER, Hayley L; SLATER, Louise; LEWIS, Anitta Antes; KON, Onn M; HOLGATE, Stephen T; DAVIES, Donna E; KOTENKO, Sergei V; PAPI, Alberto; JOHNSTON, Sebastian L. Role of deficient type III interferon- $\lambda$ production in asthma exacerbations. Nature Medicine, v.12, p. 1023-1026, 2006.

CORDER, E. H; SAUNDERS, A. M; RISCH, N. J. STRITTMATTER, W. J; Schemechel, D. E; GASKELL, P. C; RIMMLER, J. B; LOCKE, P. A; CONNEALLY, P. M; SCHMADER, K. E; SMALL, G. W; ROSAS, A. D; HAINES, J. L; VANCE, M. A. P. Protective effect of apolipoprotein E type 2 allele for late onset Alzheimer disease. Nature Genetics, v.7, p. 180-184, 1994.

ELLINGHAUS, David; DEGENHARDT, Frauke; BUJANDA, Luis; BUTI, Maria; ALBILLOS, AUGUSTÍN; INVERNIZZI, Pietro; FERNÁNDEZ; PRATI, Daniele;

BASELLI, Guido. Genomewide Association Study of Severe Covid-19 with Respiratory Failure. New England Journal of Medicine, v.6, n.17, 2020.

FELDMAN, Charles; ANDERSON, Ronald. Cigarette smoking and mechanisms of susceptibility to infections of the respiratory tract and other organ systems. Journ. of infect. v. 67, n.3, p.169-184, 2013.

GAO, Qijun; HU, Yingfu; DAI, Zhiguo; XIAO, Feng; WANG, Jing; WU, Jing. The epidemiological characteristics of 2019 novel coronavirus diseases (COVID-19) in Jingmen, Hubei, China. Medicine, v.99, n.23, 2020.

GOLDBERG, Anna Carla; RIZZO, Luiz Vicente. Estrutura e função do MHC - Apresentação de antígenos. Einstein, v.13, n.1, 2015.

GOUDOURIS, Ekaterini S. Diagnóstico laboratorial de COVID-19. J. Pediatr., v.97, n.1, p. 7-12, 2021.

GRANDY, Giuseppe; VOLZ, Carlos G. Terán; MARTÍNEZ, Alejandro; VOLZ, Anna. Covid-19, una mirada desde la pediatría. Gac. Med. Bol., v.43, n.1, 2020.

GUILLON, Patrice; CLÉMENT, Monique; SÉBILLE, Véronique; RIVAIN, Jean Gérard; CHOU, Chih Fong; CLOUET, Nathalie Ruvoen; PENDU, Jacques Le. Inhibition of the interaction between the SARSCoV Spike protein and its cellular receptor by anti-histo-blood group antibodies. Glycobiology, v.18, n.12, p. 1085-1093, 2008.

INCA. INCA divulga estudo inédito sobre Covid-19 que pode auxiliar no desenvolvimento de vacinas mais eficientes. 2021. Disponível em < https://www.inca.gov.br/es/node/4591 >. Acesso em: 22 de maio de 2021.

JAILLON, Sébastien. BERTHENET, Kevin. GARLANDA, Cecilia. Dimorfismo sexual na imunidade inata. Avaliações clínicas em alergia e imunologia. SprigerLink, v.56, p. 308-321, 2019.

JENKINS, P. VINCE; DONNEL, JAMES S. ABO blood group determines plasma von Willebrand factor levels: a biologic function after all?. Transfusion, v.46, n.10, p. 1836-1844, 2006.

KAWAHARA, Lucas Tokio; COSTA, Isabela Bispo Santos da Silva; BARROS, Cecília Chie Sakaguchi; ALMEIDA, Gabriel Coelho; BITTAR, Cristina Salvadori; RIZK, Stephanie Itala; TESTA, Laura; MONIZ, Camila Motta Venchiarutti; PEREIRA, Juliana; OLIVEIRA, Gláucia Maria Moraes; DIZ, Maria Del Pilar Estevez; GUIMARÃES, Patria Oliveira; PINTO, Ibraim Masciarelli; FILHO, Roberto Kalil; HAJJAR, 
Ludhmila Abrahão; HOFF, Paulo M. Cancer and Cardiovascular Diseases during the COVID-19 Pandemic. Arq. Bras. Cardiol., v.115, n.3, 2020.

KLEIN, Sabra L.; MARRIOTT, Ian.; FISH, Eleanor N. Sex-based differences in immune function and responses to vaccination. Transactions of The Royal Society of Tropical Medicine and Hygiene, v.109, n.1, p. 9-15, 2015.

KUO, Chia Ling; PILLING, Luke C; ATKINS, Janice L; MASOLI, Jane A. H; DELGADO, João; KUCHEL, George A; MELZER, David. ApoE e4e4 Genotype and Mortality With COVID-19 in UK Biobank. J. Gerontol A. Biol. Sci. Med. Sci., v.75, n.9, p. 1801-1803, 2020.

LUKASSEN, Soeren; CHUA, Robert Lorenz; TREFZER, Timo; KAHN, Nicolas C.; SCHNEIDER, Marc A.; MULEY, Thomas; WINTER, Hauke; MEISTER, Michael; VEITH, Carmen; BOOTS, Agnes W.; HENNING, Bianca P; KREUTER, Michael; CONRAD, Christian; EILS, Roland. SARS-CoV-2 receptor ACE2 and TMPRSS2 are primarily expressed in bronchial transient secretory cells. Embo Jour., v.39, 2020.

MACIEL, Ethel Leonor; JABOR, Pablo; JÚNIOR, Etereldes Goncalves; SÁ, Ricardo Tristão; LIMA, Rita de Cássia Duarte; SANTOS, Bárbara Reis; LIRA, Pablo; Bussinguer, Elda Coelho Azevedo; Zandonade. Mortalidad en personas ingresados en el hospital por COVID-19 en Espírito Santo, Brasil, 2020. Epidemiol. Serv. Saúde, v.29, n.4, 2020.

MACINKO, James; WOOLLEY, Natalia Oliveira; SEIXAS, Brayan V.; ANDRADE, Fabiola Bof; COSTA, Maria Fernanda Lima. Procura por atendimento médico devido a sintomas relacionados à COVID19 e cancelamento de consultas médicas em função da epidemia entre adultos brasileiros mais velhos: iniciativa ELSI-COVID-19. Cad. Saúde Pública, v.36, n.3, 2020.

MADDALONI, Ernesto; BUZZETTI, Raffaella. Covid-19 and diabetes mellitus: unveiling the interaction oftwo pandemics. Diabetes Metab. Res. Rev., v.36, 2020.

MOREIRA, Rafael da Silveira. COVID-19: unidades de terapia intensiva, ventiladores mecânicos e perfis latentes de mortalidade associados à letalidade no Brasil. Cad. Saúde Pública, v.36, n.5, 2020.

NISHIGA, Mesataka; WANG, Dao Wen; HAN, Yaling; LEWIS, David B; WU, Joseph C. COVID-19 and cardiovascular disease: from basic mechanisms to clinical perspectives. Nature Reviews Cardiology, v.17, p. $543-558,2020$.

NSANZABERA, Charles. Health Promotion to Reduce Hypertension Patients' Vulnerability to Coronavirus Disease-19 (COVID-19). Int. J. Cardiovasc. Sci., v. 34, n.1, 2021.

ORELLANA, Josem Douglas Yamall; CUNHA, Geraldo Marcelo; MARRERO, Lihsieh; HORTA, Bernardo Lessa; LEITE, Iuri Costa. Explosión de la mortalidad en el epicentro amazónico de la epidemia de COVID-19. Cadernos de Saúde, v.36, n.7, 2020.

OUDIT, G.Y; KASSIRI, Z; JIANG, C; LIU, P. P; PUTANEN, S. M; PENNINGER, J. M; BUTANY, J. SARS-coronavirus modulation of myocardial ACE2 expression and inflammation in patients with SARS. European Journ. of Clinical Invest. v. 39, n.7, p. 618-625, 2009.

PÉREZ, Gema A.; CORDERO, Camila R.; AVENDAÑO, Luis Fidel C. Otro desafío de la naturaleza: el nuevo coronavirus virología y fisiopatología del SARS-COV-2 / Another challenge of nature: the new 
coronavirus virology and physiopathology of SARS-CoV-2. Neumologia Pediatria, v.15, n.2, p.301-307, 2020.

REIS, Paulo Eduardo Ocke; LIMA, Marcos Cesar Braga. Podemos atuar preventivamente para evitar que os pacientes portadores de COVID-19 evoluam de forma mais grave?. Jornal Vascular Brasileiro, v.19, 2020.

RONCO, Claudio; REIS, Thiago; SYED, Faeq Husain. Management of acute kidney injury in patients with COVID-19. The Lancelet, v.8, n.7, p. 738-742, 2020.

SALES, Maria Penha Uchoa; ARAÚJO, Alberto José; GODOY, Irma; PEREIRA, Luiz Fernando Ferreira; CASTELlANO, Maria Vera Cruz Oliveira; TANNI, Suzana Erico; ALMEIDA, Adriana Ávila; CHATKIN, Gustavo; SILVA, Luiz Carlos Côrrea; GONÇALVES, Cristina Maria Cantarino; BOTELHO, Clóvis; SANTOS, Ubiratan Paula; VIEGAS, Carlos Alberto de Assis; SESTELO, Maristela Rodrigues; MEIRELES, Ricardo Henrique Sampaio; CORREA, Paulo César Rodrigues Pinto; OLIVEIRA, Maria Eunice Moraes; REICHERT, Jonatas; LIMA, Mariana Silva; SILVA, Celso Antonio Rodrigues. J. bras. pneumol., v.45, n.3, 2019.

SANTOS, Jefferson Pereira Caldas; SIQUEIRA, Alexandre San Pedro; PRAÇA, Heitor Levy Ferreira; Albuquerque, Hermano Gomes. Vulnerabilidade a formas graves de COVID-19: uma análise intermunicipal na cidade do Rio de Janeiro, Brasil. Cad. Saúde Pública, v.36, n.5, 2020.

SILVA, Cayo Cesar; CARVALHO, Camilla Marcelle Ozoroio; LIMA, Denis Costa; COSTA, Emmanuela Santos; ANDRADE, Victória Maria Beltrão; TENORIO, Bruno Mendes; BRITTO, Diana Babini Lapa de Albuquerque; TENORIO, Fernanda Chagas Angelo Mendes. Covid-19: Aspectos da origem, fisiopatologia, imunologia e tratamento: uma revisão narrativa. Rev. Eletrôn. Acervo Saúde, v. 13, n.3, 2021.

SIMONNET, Arthur; CHETBOUN, Mikael; POISSY, Julien; RAVERDY, Violeta; NOULETTE, Jerome; DUHAMEL, Alain; LABREUCHE, Julien; MATHIEU, Daniel; PATTOU, Francois; JOURDAIN, Merce. High Prevalence of Obesity in Severe Acute Respiratory Syndrome Coronavirus-2 (SARS-CoV-2) Requiring Invasive Mechanical Ventilation. The Obesity Society, v.28, n.7, 2020.

SING, C. F; DAVIGNON, J. Role of the apolipoprotein E polymorphism in determining normal plasma lipid and lipoprotein variation. Am. J. Hum. Genet., v.37, n.2, p. 268-285, 1985.

SOUZA, Larissa Gonçalves; RANDOW, Raquel; SIVIERO, Pamila Cristina Lima. Reflections about the COVID-19: differentials by sex and age. Com. Ciências da Saúde, v.31, n.1, p.75-83, 2020.

SOSIBO; Aubrey Mbulelo. KHATHI, Andile. Pre-diabetes and COVID-19, could we be missing the silent killer?. Sage Journ., v.246, n.4, 2021.

SUÁREZ, Jennifer Carolina Gutierrez; URREGO, Carmen Cecilia Almonacid; ROJAS, Edith del Carmen Hernández; ZERÓN, Hugo Mendieta. Prognostic value of biochemical markers in patients with COVID19. Nova, v.18, n.35, 2021.

TAGARRO, Alfredo; EPALZA, Cristina; SANTOS, Mar. SANTAEUFEMIA, Francisco José Sanz; OTHEO, Enrique; MORALEDA, Cinta; CALVO, Cristina. Screening and Severity of Coronavirus Disease 2019 (COVID-19) in Children in Madrid, Spain. JAMA Pediatr., v.175, n. 3, p.316-317, 2021. 
TANG, Ning; LI, Dengju; WANG, Xiong; SUN, Ziyong. Abnormal coagulation parameters are associated with poor prognosis in patients with novel coronavirus pneumonia. J. Thromb. Haemost. v.18, n.4, p.844$847,2020$.

TAVARES, Caio de Assis Moura; SILVA, Thiago Junqueira Avelino; CARDOZO, Francisco Akira Malta; FERNANDES, Juliana Ruiz; GIRARDI, Adriana Castello Costa; FILHO, Wilson Jacob. ACE2 Expression and Risk Factors for COVID-19 Severity in Patients with Advanced Age. Arq. Bras. Cardiol., v.115, n.5, 2020.

TAYLOR, Graig J; BOLTON, Eleanor M; BRADLEY, J. Andrew. Immunological considerations for embryonic and induced pluripotent stem cell banking. Philos Trans. R. Soc. Lond. B. Biol. Sci., v.366, n.1575, p. 2312-2322, 2011.

VALLADARES, Adriana Moreno; PERDOMO, Álvaro E. Cartagena; PABÓN, Guilhermo Mora. Apolipoprotein E and cardiovascular disease. Rev. Fac. Med., v.54, n.1, 2006.

VÁZQUEZ, Javier Correa; RIPOLL, Diego Redolar. Epidemiological Data From the COVID-19 Outbreak in Spain for the Promotion of Tobacco Smoking Cessation Policies. SAGE Journal, v.13, p. 1-2, 2020.

VELAVAN, Thirumalaisamy P.; MEYER, Christian G. The COVID-19 epidemic. Trop Med. Int. Health, v.25, n.3, p. 278-280, 2020.

VIEIRA, Luisane Maria Falci; EMERY, Eduardo; ANDRIOLO, Adagmar. COVID-19: diagnóstico laboratorial para médicos. Um artigo de atualização. São Paulo Med. J. v, 138, n.3, 2020.

WAN, Yushun; SHANG, Jian; Graham, Rachel; BARIC, Ralph S; LI, FANG. Receptor Recognition by the Novel Coronavirus from Wuhan: an Analysis Based on Decade-Long Structural Studies of SARS Coronavirus. Journ. of Virol., v.94, n.7, p. 1-9, 2020.

WANG, Fang; HUANG Shujia; GAO, Rongsui; ZHOU, Yuwen; LAI, Changxiang; LI, Zhichao; XIAN, Wenjie; QIAN, Xiaobo; LI, Zhiyu; HUANG, Yushan; TANG, Qiyuan; LIU, Panhong; CHEN, Ruikun; LIU, Rong; LI, Xuan; TONG, Xin; ZHOU, Xuan; BAI, Yong; DUAN, Gang; ZHANG, Tao; XU, Xun; WANG, Jian; YANG, Huanming; LIU, Siyang; HE, Qing; JIN, Xin; LIU, LEI. Initial Whole Genome Sequencing and Analysis of the Host Genetic Contribution to COVID-19 Severity and Susceptibility. MedRxiv, 2020.

WU, O; BAYOUMI, N; VICKERS, MA; CLARK, P. ABO $(\mathrm{H})$ blood groups and vascular disease: a systematic review and meta-analysis. Journ. of thrombo. and haemost., v.6, n.1, p. 62-69, 2008.

WU, Zunyou; MCGOOGAN, Jennifer M. Characteristics of and Important Lessons From the Coronavirus Disease 2019 (COVID-19) Outbreak in China. Jama, v.323, n.13, p.1239-1242, 2020.

XU, Hao; ZHONG, Liang; DENG, Jiaxin; PENG, Jiakuan; DAN, Hongxia; ZENG, Xin; LI, TAIWEN; CHEN, Qianming. High expression of ACE2 receptor of 2019-nCoV on the epithelial cells of oral mucosa. Interna. Journ. of Oral Scienc., v.12, n.8, 2020.

YUKI, Koichi; FUJIOGI, Miho; KOUTSOGIANNAKI, Sophia. COVID-19 pathophysiology: A review. Clinical Immunology, v.215, 2020. 
ZIETZ, Michael; ZUCKER, Jason; TATONETTI, Nicholas P. Testing the association between blood type and COVID-19 infection, intubation, and death. MedRxiv, v.3, 2020. 